ARTICLE

DOI: $10.1038 / s 41467-018-04572-3$

OPEN

\title{
In vivo screening identifies GATAD2B as a metastasis driver in KRAS-driven lung cancer
}

\author{
Caitlin L. Grzeskowiak', Samrat T. Kundu², Xiulei Mo ${ }^{3}$, Andrei A. Ivanov³, Oksana Zagorodna', Hengyu Lu (1) 1, \\ Richard H. Chapple1, Yiu Huen Tsang (1) 1, Daniela Moreno', Maribel Mosqueda ${ }^{4}$, Karina Eterovic ${ }^{4}$, \\ Jared J. Fradette ${ }^{2}$, Sumreen Ahmad ${ }^{2}$, Fengju Chen ${ }^{5}$, Zechen Chong ${ }^{6}$, Ken Chen (1) ${ }^{6}$, Chad J. Creighton ${ }^{5,6,7}$, \\ Haian Fu (1] ${ }^{3}$, Gordon B. Mills ${ }^{4}$, Don L. Gibbons ${ }^{2,8}$ \& Kenneth L. Scott ${ }^{1}$
}

Genetic aberrations driving pro-oncogenic and pro-metastatic activity remain an elusive target in the quest of precision oncology. To identify such drivers, we use an animal model of KRAS-mutant lung adenocarcinoma to perform an in vivo functional screen of 217 genetic aberrations selected from lung cancer genomics datasets. We identify 28 genes whose expression promoted tumor metastasis to the lung in mice. We employ two tools for examining the KRAS-dependence of genes identified from our screen: 1) a human lung cell model containing a regulatable mutant KRAS allele and 2) a lentiviral system permitting co-expression of DNA-barcoded cDNAs with Cre recombinase to activate a mutant KRAS allele in the lungs of mice. Mechanistic evaluation of one gene, GATAD2B, illuminates its role as a dual activity gene, promoting both pro-tumorigenic and pro-metastatic activities in KRAS-mutant lung cancer through interaction with $c-M Y C$ and hyperactivation of the c-MYC pathway.

\footnotetext{
${ }^{1}$ Department of Molecular and Human Genetics, Baylor College of Medicine, Houston, TX 77030, USA. ${ }^{2}$ Department of Thoracic/Head and Neck Medical Oncology, The University of Texas MD Anderson Cancer Center, Houston, TX 77030, USA. ${ }^{3}$ Department of Pharmacology and Emory Chemical Biology Discovery Center, Emory University School of Medicine, Atlanta, GA 30322, USA. ${ }^{4}$ Department of Systems Biology, University of Texas MD Anderson Cancer Center, Houston, TX 77030, USA. ${ }^{5}$ Dan L. Duncan Cancer Center, Baylor College of Medicine, Houston, TX 77030, USA. ${ }^{6}$ Department of Bioinformatics and Computational Biology, The University of Texas MD Anderson Cancer Center, Houston, TX 77030, USA. ${ }^{7}$ Department of Medicine, Baylor College of Medicine, Houston, TX 77030, USA. ${ }^{8}$ Department of Molecular and Cellular Oncology, University of Texas MD Anderson Cancer Center, Houston 77030, USA. These authors contributed equally: Caitlin Grzeskowiak, Samrat T. Kundu. These authors jointly supervised this work: Don L. Gibbons, Kenneth L. Scott. Deceased: Kenneth L. Scott. Correspondence and requests for materials should be addressed to D.L.G. (email: dlgibbon@mdanderson.org)
} 
$\mathrm{N}$ on-small cell lung cancer (NSCLC) is the leading cause of cancer mortality in the United States, primarily due to the development of metastatic disease ${ }^{1}$. Recent progress in treating lung cancer has come from identifying patient subpopulations with identifiable oncogenic mutations that can be targeted with small molecule inhibitors (e.g., erlotinib and crizotinib for EGFR-driven and EML4-ALK-driven tumors, respectively $)^{2,3}$. Unfortunately, the majority of lung cancer cases are driven by unknown genetic events or mutations in genes such as $K R A S$ (30\% of patients) for which there are no selective therapeutics ${ }^{4}$.

$K R A S$-driven lung cancers are not targetable by currently approved therapies and represent a particularly aggressive form of NSCLC $^{5}$. Regional or distant metastases are thought to form often and early in KRAS-driven adenocarcinoma leading to high mortality $^{6,7}$. Subgroups have been identified in KRAS-mutant NSCLC based upon co-mutations that enhance or modulate $K R A S$ tumorigenicity and disease progression, providing biological and molecular context for personalized and targetable treatments for KRAS mutant patients ${ }^{8}$. New approaches aimed at personalized therapeutic strategies are critical for these patients, as identifying targets downstream of or that work in conjunction with KRAS offer the most promising opportunities to exploit therapeutic vulnerabilities. Therefore, systematic functional characterization of lung cancer genome datasets is needed. The Cancer Genome Atlas (TCGA) and others have generated a compendium of genomic aberrations in lung cancer with the goal of identifying the most promising drug targets and diagnostic biomarkers ${ }^{9}$. The challenge now is to distinguish the subsets of functional oncogenic and metastatic driver aberrations from passenger mutations that do not offer therapeutic opportunities.

While RNA interference (RNAi)-based and CRISPR/Cas9based genetic screening platforms have successfully identified new tumor suppressor genes and other genetic vulnerabilities in cancer, several recent studies reveal a complementary approach through developing scalable gain-of-function screening systems for validating over-expressed or mutationally activated oncogenes that, as a class, have served as successful therapeutic targets to date. For example, we previously reported a multi-level functional assessment platform, High-Throughput Mutagenesis and Molecular Barcoding (HiTTMoB), which has identified novel variants of known oncogenes ${ }^{10}$, as well as elucidating novel drivers of pancreatic ductal adenocarcinoma ${ }^{11}$. Here we report an adaptation of this platform to identify genetic drivers that synergize with mutant KRAS to advance tumor progression and metastasis in lung adenocarcinoma. In vivo functional screening of a gene library informed by oncogenomics-guided integration of mutant $K R A S$-specific mouse and human gene signatures reveal several genes whose expression promote tumor growth and/or metastasis, as outlined here and in the companion paper. Among those genes, functional characterization of GATAD2B illuminates its role as a potent driver of tumor growth and metastasis in KRASdriven lung cancers. We further show here that high GATAD2B expression correlates with worsened outcomes in lung cancer patients and cooperates with KRAS to promote gain-of-function pro-oncogenic and pro-metastatic transcriptional programs including $M Y C$ to mediate cell invasion in vitro and tumor progression in vivo.

\section{Results}

In vivo screening for drivers of lung cancer metastasis. We and others have shown that mouse and human tumors of diverse tissue lineages sustain orthologous genomic aberrations that can function as bona fide cancer driver events ${ }^{12-16}$. These observations prompted us to utilize cross-species, integrative analyses to biologically filter and prioritize TCGA data to define a gene list enriched for lung cancer drivers. To do this, we first leveraged published transcriptome comparisons of spontaneous lung adenocarcinomas and metastases isolated from $\mathrm{Kras}^{\mathrm{LA1} /+}$, $p 53^{R 172 H \Delta G /+}$ mice $^{17}$ in order to select 615 genes $(p<0.01$, paired t-test) up-regulated in Kras ${ }^{L A 1 /+}, p 53^{R 172 H \Delta G /+}$ metastases compared to primary tumors. We next intersected these data with transcriptome comparisons of non- and strongly metastatic murine $393 \mathrm{P}$ and 344SQ syngeneic tumors, respectively, grown from cell lines isolated from spontaneous $\mathrm{Kras}^{\mathrm{G} 12 D}, \mathrm{p} 53^{\mathrm{R} 172 \mathrm{H} \Delta G}$ tumors $^{18}$, which revealed 1220 genes expressed significantly higher in metastatic 344SQ cells vs. 393 P tumors $(p<0.01$, paired $t$-test $)^{18}$. To control for the possibility of cooperation between $K R A S^{G 12 D}$ and $p 53^{R 172 H \Delta G}$ used in our model, we compared gene expression levels of all ORFs used in our screening strategy between murine lung tumors from the KRAS $G 12 D$ and KRAS ${ }^{G 12 D}$; $p 53^{R 172 H \Delta G}$ models, and observed that less than $3 \%$ of the 225 ORFs were significantly expressed $(p<0.01$, paired $t$-test $)$ in the KRAS $S^{G 12 D} ; p 53^{R 172 H \Delta G}$ model compared to the KRAS G12D model (Supplementary Data 1). Genes found up-regulated in KRAS models were next triangulated with human copy number amplifications documented by TCGA (1.5-fold somatic amplification across $\geq 5 \%$ of 154 analyzed lung adenocarcinoma specimens). In total, this cross-species analysis generated a list of 220 overlapping genes.

Because oncogenic pathways can be similarly activated by hyperactivation mutations, an observation first discovered by well-characterized oncogenes such as PIK3CA and $A L K^{19-23}$, we integrated sequencing results by Ding and colleagues ${ }^{24}$ that focused on 623 genes with potential relationships to cancer. Filtering copy number amplifications from TCGA with the 1013 non-synonymous somatic mutations identified by their study revealed 31 amplified genes that contain at least one validated missense mutation within the 188 analyzed tumors. Combining these data with the 220 genes described above yielded a total of 251 candidate genes that include several known to play a significant role in lung cancer (e.g., IKK-BETA, KRAS, EGFR, ZEB1, TWIST1), in addition to two potent drivers (FSCN1 and HOXA1) of melanoma transformation and metastasis identified in our previous studies ${ }^{12,25}$. Of the 251 candidate genes identified, 225 were available in our collection of open reading frame (ORF) clones comprised of the Human ORFeome collection ${ }^{26}$, as well as other commercially available ORF clones. Conservation analysis was performed to reduce the potential for false negatives in our screening model and confirmed significant homology of the 225 genes between mouse and human (Supplementary Data 1). Included in the 225 ORFs were 28 wild-type ORFs representing mutated genes in Ding et. al studies (Supplementary Data 1). Each ORF was introduced into a lentiviral vector through recombination-mediated cloning. Importantly, each expression vector was uniquely tagged with a 24-nucleotide DNA barcode (Supplementary Data 1) during ORF lentiviral integration using our previously reported High-Throughput Mutagenesis and Molecular Barcoding (HiTMMoB) strategy ${ }^{11}$, providing a surrogate identifier for each associated ORF.

We next sought to examine tumor growth and metastatic activity of selected candidates by performing a pooled, in vivo screen for drivers of lung metastasis (Fig. 1). Barcoded lentiviral constructs were used to generate lentivirus in a multi-well format, followed by transduction of non-metastatic murine lung cancer cells (393 P) derived from the Kras ${ }^{L A 1 /+} ; p 53^{R 172 H \Delta G}$ genetically engineered mouse (GEM) model ${ }^{18}$ from which the candidate gene library was partially derived. Of the 225 transduced cell lines, 217 positively selected for viral integration with puromycin (Supplementary Data 1). The 217 cell lines were individually transduced to reduce biases of multi-gene interactions. While 

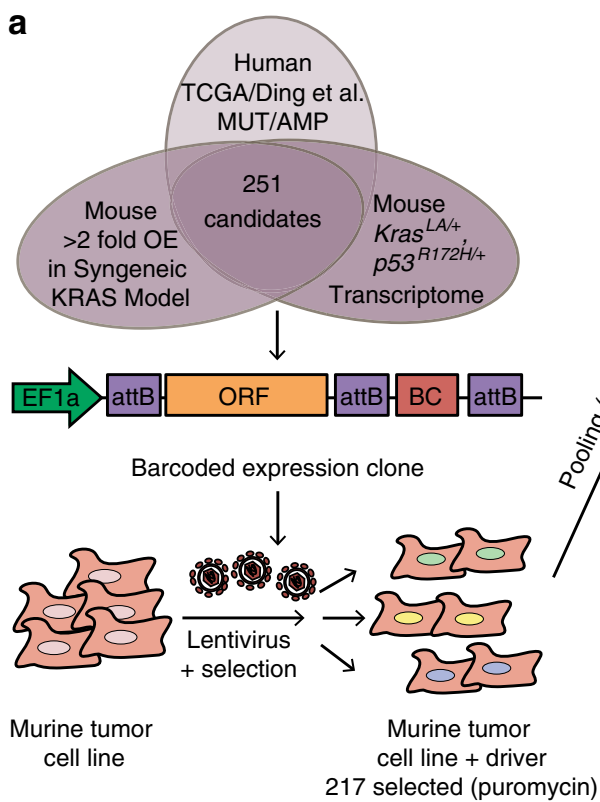

b

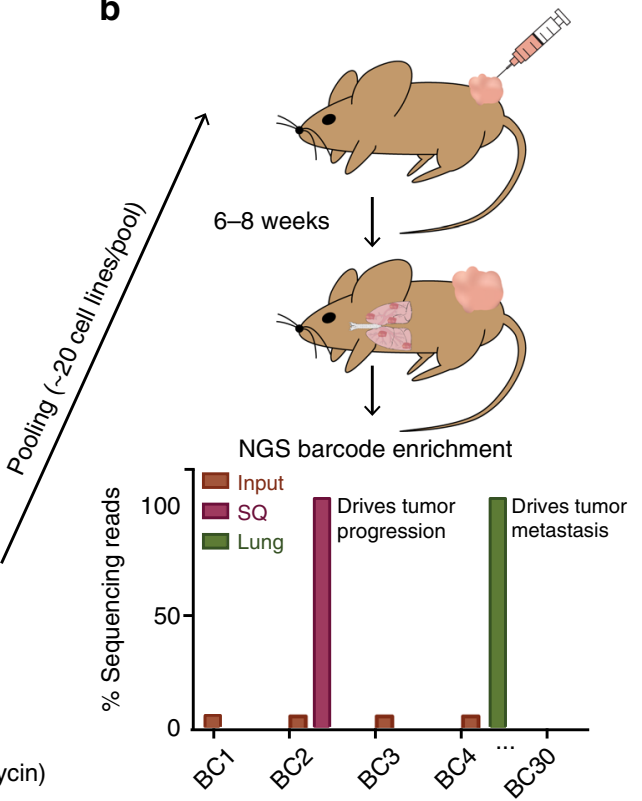

Fig. 1 In vivo functional screening for KRAS effectors driving metastasis. a Illustration of Oncogenomics informed screening of DNA barcoded candidate genes cloned by HiTMMoB and introduced into non-metastatic $393 \mathrm{P}$ murine tumor cell line via lentiviral transduction. b Individual ORF-barcoded cell lines were pooled and injected into the flanks of immunocompetent 129 Sv mice, followed by barcode enrichment analysis of injected cells (Input), subcutaneous (SQ) tumors and lung metastases (see text for details). Representative histogram illustrates positive enrichment of hypothetical driver of SQ growth (BC2) and lung metastasis (BC4). attB recombination cassettes; BC barcode. Elements of image used with permission of Patrick J. Lynch and Carl Jaffe, MD under Creative Commons Attribution 2.5 License 2006

individually transduced cell lines may limit complexity of the screening library, it does ensure expression of all potential oncogenes with a readily identifiable ORF-surrogate using the DNA barcode. The 217 cell lines, along with mCherry-expressing control cells, were pooled (average pool size $=20$ genes/pool; Supplementary Data 2) for subcutaneous (SQ) injection into the flanks of immunocompetent syngeneic $129 \mathrm{~Sv}$ mice $(N=10$ mice/ pool; $N=120$ mice total). Murine $393 \mathrm{P}$ cells develop primary SQ tumors over the course of 6-8 weeks when injected into $129 \mathrm{~Sv}$ mice and do not metastasize as parental cells or those expressing mCherry control (Supplementary Fig. 1). In contrast, lung metastases were readily observed when animals were injected with cells across multiple pools, generating a streamlined approach for rapid analysis of metastatic lesions in our screen (Supplementary Fig. 1, Supplementary Table 1).

We have observed from in vivo tumor screens that, relative to injected cells (input), tumors and metastases positively select ORF drivers and lose those with no role in progression ${ }^{10,11}$. Individual enrichment among pooled ORFs can be determined by comparing the number of individual ORF-associated barcode reads within experimental pools as a ratio of each barcode to the total number of barcode reads per NGS amplicon. For example, ORFdriven SQ tumors (barcode 2) and lung metastases (barcode 4) will be positively enriched for driver-associated barcodes and lose those with no role in progression (that is, passengers, barcode 1 and 3; Fig. 1). To determine which ORFs were positively selected in tumor and metastatic tissues, we next collected quadruplicate SQ tumor and independent metastatic lung lesions when present from SQ tumor-bearing mice (Supplementary Table 1). We quantitated ORF barcode reads from genomic DNA isolated from SQ tumors, metastases, and injected cells using next generation sequencing (NGS) as described previously ${ }^{11}$. Our assessment of ORF barcode enrichment in SQ tumors vs. input identified 21 (representing 19 unique genes) of 217 ORFs (\% of mice where $N>2$ mice analyzed, SQ BC enrichment $>$ input $=100 \times$;
Supplementary Fig. 2a and Supplementary Data 2), suggesting that these ORFs may enhance cell proliferation and/or tumor growth in $393 \mathrm{P}$ cells. Assessment of barcode enrichment in lung metastases vs. input identified 28 ORFs positively enriched in multiple $(N \geq 2)$ lung metastasis samples in two or more mice per cohort of 10 pool-injected mice, therefore $89 \%$ of cell lines (189 in total) were not present in macrometastases collected upon necropsy (Fig. 2a and Supplementary Table 1). No macrometastases were present in cohorts injected with pools 1 and 2. Notable ORFs enriched in metastatic tissues include GNAS and MYC, which were previously shown to promote KRAS-driven lung cancer metastasis 27,28 . Comparison of SQ-enriched and metastasis-enriched ORFs found 7 positively enriched in both, suggesting promotion of tumor growth and metastasis, whereas 21 ORFs were found enriched only in metastases (Supplementary Fig. 2b).

GATAD2B expression correlates with poor survival. We sought to identify progression mediators of KRAS-driven lung cancer, thus we resourced TCGA datasets to determine whether amplification and copy number-driven expression (Supplementary Fig. 3a) of the 28 metastasis-enriched genes from the screen correlated with KRAS mutation status in lung adenocarcinoma patients. We identified three genes (GATAD2B, ACVR1B, and ZC3H11A) whose overexpression significantly correlates with mutant KRAS lung adenocarcinoma vs wild-type KRAS status in patients (Supplementary Table 2). Additional mining of TCGA genomics data revealed that GATAD2B is also commonly amplified in KRAS mutant cancers in other lineages, including pancreatic ductal adenocarcinoma, where KRAS is mutationally activated in greater than $90 \%$ of cases (Fig. 2b) ${ }^{4,9,29,30}$. We queried TCGA lung adenocarcinoma patient datasets fully annotated for gene expression (TCGA-provisional as queried using [http://www.cbioportal.org/] $)^{31}$. Through evaluating 

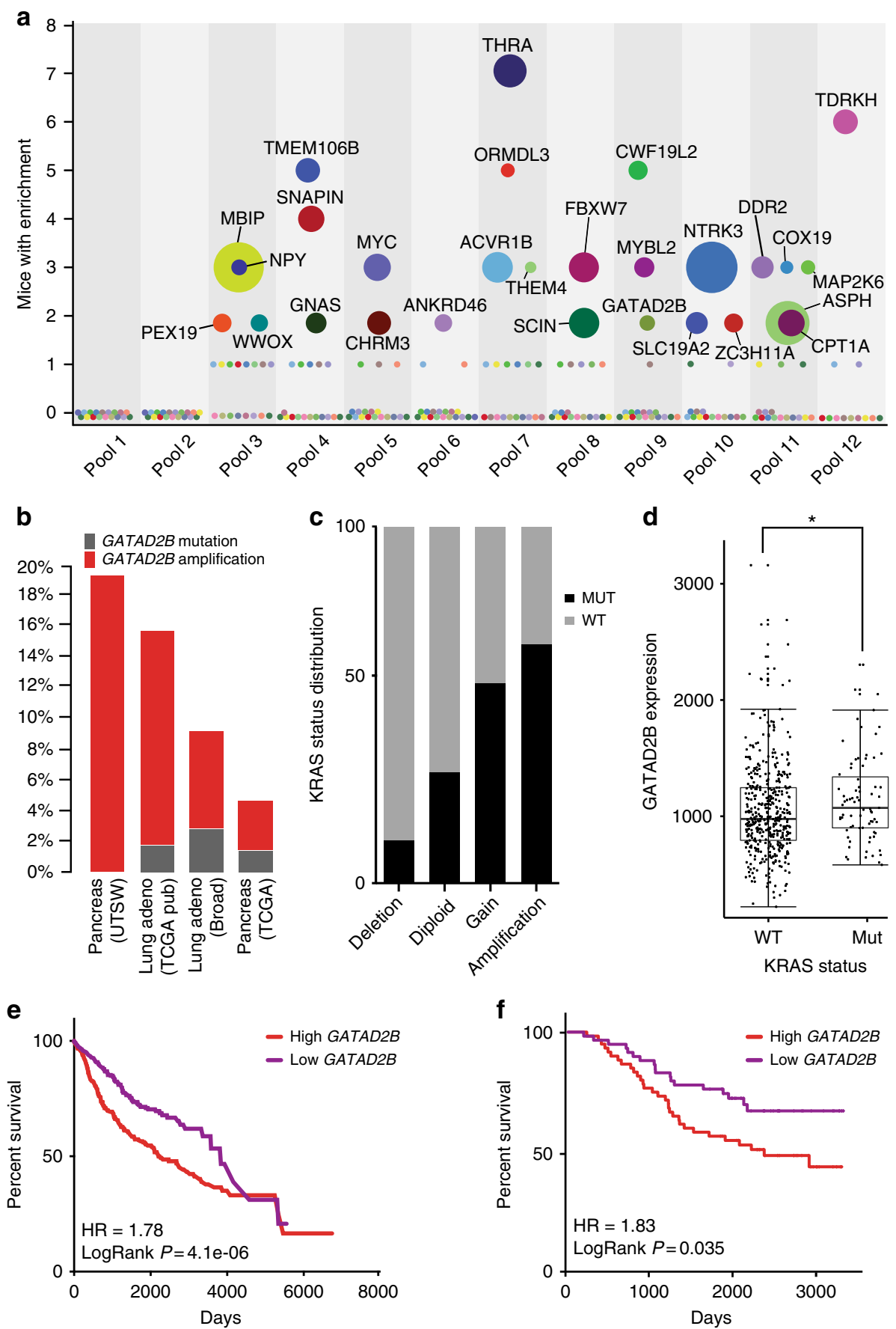

Fig. 2 In vivo metastasis driver screening identifies GATAD2B. a Barcode sequencing analysis identifies 28 gene candidates enriched in lung metastases present in at least two mice per pool cohort. Individual genes are color-coded. Circumference of circle correlates with average enrichment in lung tissue vs. input (see Supplementary Data 1). b GATAD2B amplification and mutation across KRAS-driven cancers as reported by TCGA and others ${ }^{4,9,29}$. c Frequency of GATAD2B putative GISTIC copy number status in KRAS mutant $(n=75)$ vs. WT $(n=437)$ patients, (chi-square statistic $46.11083 D F$, ${ }^{\star} p$-value $<0.00001$. d Expression levels of GATAD2B in KRAS mutant (RNAseq expression 980.8) vs. wild type (1072.0) lung adenocarcinoma patients $(n=517$, Wilcoxon rank-sum test, $p<0.0350)$. e, $\mathbf{f}$ Increased GATAD2B expression correlates with worse patient prognosis in (e) lung adenocarcinoma patients $^{32}$ and (f) patients with KRAS-driven lung adenocarcinomas ${ }^{34}$

GATAD2B copy number status in KRAS mutant $(n=75)$ vs. WT $(n=437)$ patients, we identified a significant distribution of GATAD2B putative GISTIC copy number gain and amplification in KRAS mutant compared to wild-type, (chi-square 46.1108, 3 DF, $p<0.00001$; Fig. 2c). Finally, consistent with the previous NSCLC dataset (Supplementary Table 2), GATAD2B copynumber driven expression $\left({ }^{* * * *} p<0.0001, t\right.$-test, Supplementary
Fig. 3a) was higher in KRAS mutant compared to wild type lung adenocarcinomas $(n=517$ patients, Wilcoxon rank-sum, $p<$ 0.0350, Fig. 2d).

Survival analysis of 1145 lung cancer patients ${ }^{32,33}$, indicated worse overall outcome for those whose tumors expressed high vs. low levels of $G A T A D 2 B(\mathrm{HR}=1.49, P<2.6 \mathrm{E}-6, \log$ rank test; Supplementary Fig. $3 \mathrm{~b}$ ), a finding that was even more significant 
among patients with adenocarcinoma $(\mathrm{HR}=1.78, P<4.1 \mathrm{e}-06$, $\log$ rank test; Fig. 2e). We found no correlation between GATAD2B expression and outcome among patients with squamous cell carcinoma, a lung cancer subtype rarely associated with mutationally activated KRAS ( $\mathrm{HR}=1$; Supplementary Fig. 3c) ${ }^{32}$. Importantly, elevated GATAD $2 B$ expression correlated with poor patient survival specifically in KRAS-driven lung cancer as determined by Rousseaux et al., further suggesting that GATAD2B may enhance KRAS-driven tumor growth in lung adenocarcinoma patients $(\mathrm{HR}=1.83, P=0.035$, log rank test; Fig. $2 \mathrm{f})^{33,34}$.

GATAD2B promotes pro-tumorigenic and pro-metastatic activity. To functionally examine the effect of increased GATAD2B expression in the context of activated KRAS, we constructed a primary lung cell model to permit regulated expression of $K R A S^{G 12 D}$. To do this, we leveraged a non-transformed, immortalized, human primary bronchial epithelial cell line (HBEC; hTert, CDK4, TP53 knockdown) that remains anchorage dependent and does not develop tumors when implanted into mice ${ }^{35}$. We next modified these cells by stably integrating a regulatable $K R A S^{G 12 D}$ allele, $i K R A S^{G 12 D 11}$, such that expression of mutant KRAS at physiological levels is activated upon addition of doxycycline (Dox $500 \mathrm{ng} / \mathrm{ml}$; Fig. 3a). RNA profiling of HBEC-iKRAS $S^{G 12 D}$ and HBEC- $i K R A S^{W T}$ cells revealed widespread changes in gene expression for HBEC cells harboring the activated KRAS ${ }^{G 12 D}$ allele in the presence of Dox when compared to the wild-type KRAS allele (2869 gene probes; fold change $>1.5$-fold for on-Dox vs. off-Dox, $p<0.01$, $t$-test; Fig. $3 \mathrm{~b}$ and Supplementary Data 3). Of the KRAS ${ }^{G 12 D_{-}}$ induced genetic changes, the Molecular Signatures Database (MSigDB; [http://software.broadinstitute.org/gsea/msigdb/index. jsp]) identified the oncogenic $R A S$ signature as a top-enriched gene set $(p=2.41 \mathrm{e}-92$, one-sided Fisher's exact test; Supplementary Data 4). Upregulation of RAS signaling in Dox-treated HBEC-iKRAS ${ }^{G 12 D}$ cells was also supported by a significant overlap with a KRAS signature previously characterized by Singh et al. $\left(p>2.1^{\mathrm{e}-11}\right)^{36}$.

We next used the HBEC-iKRASG12D cell model to assess the effect of expressing constitutive GATAD2B in the presence and absence of KRASG12D induction by doxycycline. We first applied HBEC-iKRAS ${ }^{G 12 D}$ cells to in vitro Matrigel cell invasion assays, which indicated that induction of $K R A S^{G 12 D}$ alone led to a modest increase in invasive capacity (Fig. $3 c ; p<0.0226, t$-test) compared to Off-Dox control cells. Stable expression of GATAD2B had no effect on the ability of HBEC-iKRAS ${ }^{G 12 D}$ cells to invade matrix in the absence of Dox; however, simultaneous induction of KRAS ${ }^{G 12 D}$ expression in the setting of constitutive GATAD2B over-expression led to a synergistic effect on cell invasion $\left({ }^{* *} p<0.01,2\right.$ way- ANOVA; Fig. $\left.3 c\right)$. The observed increase in cell invasion in the $22 \mathrm{~h}$ assay was not due to an increase in GATAD2B-mediated cell proliferation, as proliferation assays indicated no difference between GFP-expressing and GATAD2B-expressing cells in the presence of Dox over a 4day period (Supplementary Fig. 4a), however; we did observe a marked decrease in proliferation upon addition of $K R A S^{G 12 D}$, indicating an oncogene-induced senescent phenotype in these non-transformed cell lines, which was confirmed by staining for beta-galactosidase (Supplementary Fig. $4 \mathrm{~b}$ ).

We next examined whether GATAD2B expression would influence HBEC-iKRAS ${ }^{G 12 D}$ tumor growth and/or metastasis when subcutaneously implanted into the flanks of athymic mice. GFP control-expressing HBEC-iKRAS ${ }^{G 12 D}$ cells did not form tumors in mice maintained off Dox chow (Fig. 3d, orange line, $N=10$ ), and randomizing mice injected with these cells onto a Dox diet led to small palpable tumors (average $\sim 200 \mathrm{~mm}^{3}$ ) that did not progress beyond their small size over the $\sim 6$ month duration of the tumor assay (Fig. 3d, blue line, $N=10$ ). Similarly, HBEC-iKRAS G12D stably expressing GATAD2B did not develop tumors in the absence of Dox (black line, $N=4$ ); however, mice injected with these same cells developed robustly growing tumors when fed a Dox diet to turn on $K R A S^{G 12 D}$ expression (red, $N=8$ and purple lines $N=2$ ). To determine whether GATAD2B-expressing HBEC- $i K R A S^{G 12 D}$ tumors (on Dox) require continued KRASG12D expression for tumor maintenance, a subset of tumor-bearing mice were transitioned to an Off-Dox diet (On $>$ Off, purple line). These tumors regressed to baseline (compared to HBEC-iKRASG12D_GFP on Dox) thus indicating tumor growth dependence on activated KRAS, and transitioning animals back onto a Dox diet led to tumor re-development (Fig. 3d, purple line). Similarly, the non-progressive GFP control-expressing HBEC-iKRAS ${ }^{G 12 D}$ tumors that developed on doxycycline also regressed when Dox was removed (green line, $N=6$ ). Because GATAD2B has a known role in chromatin modification and transcriptional control, ${ }^{37-39}$ we verified that GATAD2B expression neither enhanced KRAS ${ }^{G 12 D}$ expression from the inducible promoter nor influenced MAPK signaling as assessed through immunoblot analysis of phospho-ERK (T202/Y204) in the presence or absence of Dox (Supplementary Fig. 4c-d). Taken overall, these in vivo data demonstrate that KRAS ${ }^{G 12 D}$ expression is necessary for tumor formation, but insufficient to support progressive tumor growth, while combined expression with GATAD2B drives progressive tumor growth that is dependent on continued KRAS G12D signaling.

Immunohistochemical analysis of HBEC- $i K R A S^{G 12 D_{-}}$ GATAD2B SQ tumors (On Dox) verified increased levels of GATAD2B expression, which was consistent with RT-qPCR analysis of the GATAD2B transcript compared to GFP-expressing tumors (Supplementary Fig. 4e), and proliferation as determined through staining for KI-67 (Fig. 3e). Consistent with the ability of GATAD2B to drive in vivo metastasis of $393 \mathrm{P}$ cells in our primary screen, necropsies of tumor-bearing HBEC-iKRAS ${ }^{G 12 D_{-}}$ GATAD2B mice revealed distant metastases $(N=4 / 5$ mice examined) to multiple lymph nodes and other organ sites (Supplementary Fig. 4f). Isolation of genomic DNA from primary tumor followed by barcode sequencing revealed robust enrichment of GATAD2B-associated barcode as expected for SQ tumor site (Supplementary Fig. $4 \mathrm{~g}$ ) and metastasis-infiltrated tissues originating from GATAD2B-transduced HBEC-iKRAS ${ }^{G 12 D}$ cells (Fig. 3f). In contrast, multiple control tissues analyzed from mice bearing HBEC- $i K R A S^{G 12} D_{-}$GFP primary tumors exhibited complete absence of GFP-associated barcode, which is consistent with the inability of HBEC-iKRASG12D cells to metastasize.

We sought to determine whether GATAD2B is required for oncogenic growth of KRAS-mutant human NSCLC. To do this, we first validated the efficacy of two independent shRNA hairpins (sh-3 and sh-5) targeting GATAD2B compared to non-targeting vector (shNT) (Supplementary Fig. 5). We next assessed the effect of GATAD2B depletion on anchorage-independent colony growth by five NSCLC cell lines with and without mutant KRAS: NCI-H23, A549, CALU1, NCI-H1437, and NCI-H1568. In all three KRAS mutant lines (H23, A549, CALU1), depletion of GATAD2B using both GATAD2B shRNAs robustly attenuated colony formation compared to shNT control, while no significant differences were observed in KRAS wild-type lines (H1437 and H1568) (Fig. 3g). Taken together, these data support the role of GATAD $2 B$ as a KRAS-dependent driver of oncogenesis and metastasis.

GATAD2B enhances tumor growth in vivo. While culture-based studies using engineered cell models such as HBEC-iKRAS ${ }^{G 12 D}$ are genetically tractable, they fail to recapitulate the microenvironment critical for the growth of human cancer cells. 
a
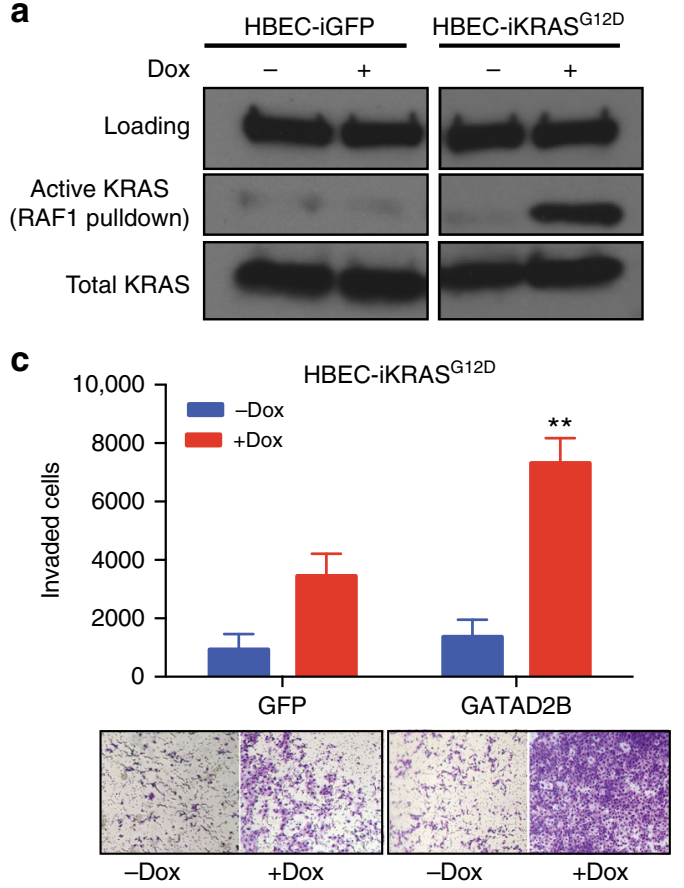

e

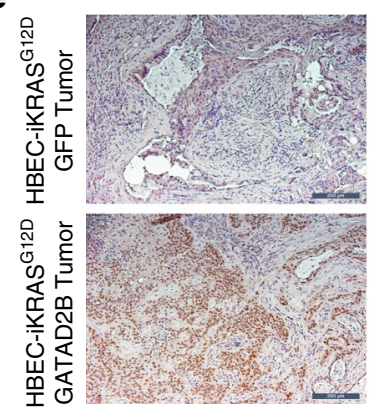

GATAD2B

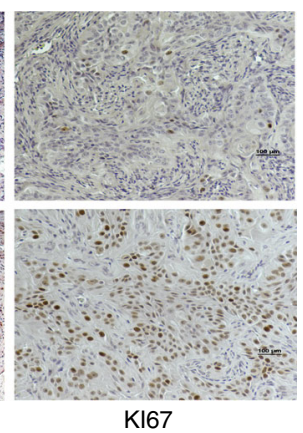

b

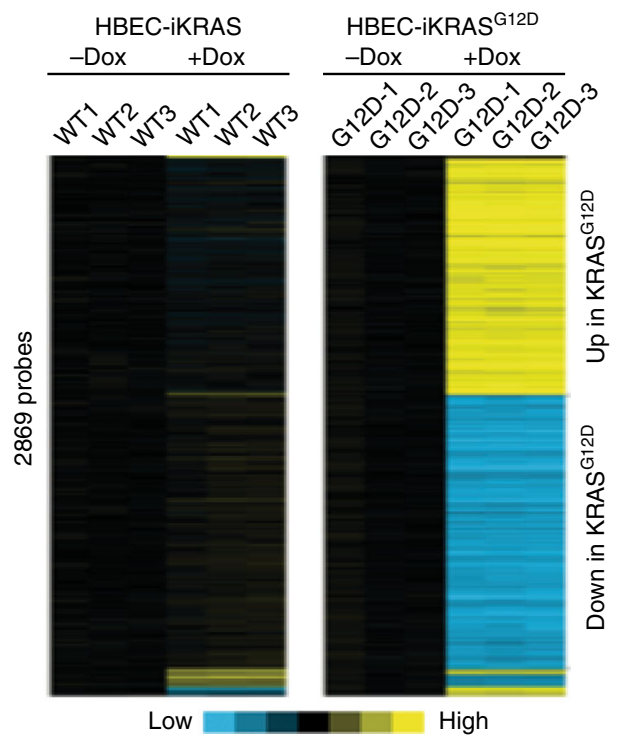

d
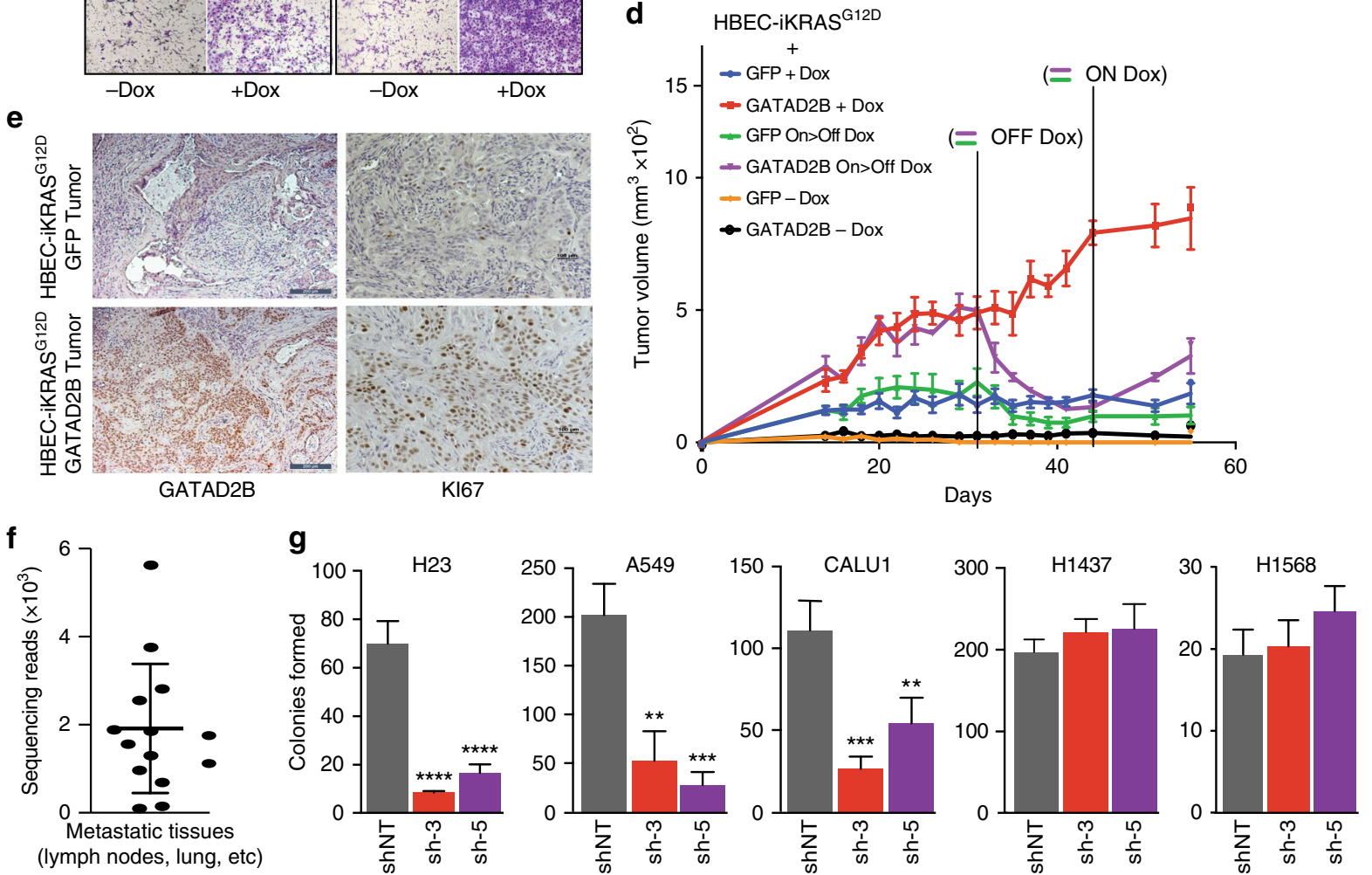

Fig. 3 GATAD2B drives KRAS-dependent HBEC tumor growth and metastasis. a Immunoblot analysis of total and activated KRAS (via RAF1 pulldown assay) extracted from HBEC cells expressing GFP or KRASG12D in the presence or absence of Dox. b Differentially expressed mRNAs in HBECs engineered with Dox-inducible alleles encoding wild-type (WT) or mutationally activated (G12D) KRAS ( $n=3$ each) in the presence or absence of Dox. c Transwell invasion assay of HBEC-iKRASG12D cells stably expressing GFP or GATAD2B in the presence or absence of Dox ( ${ }^{\star \star} p<0.01,2$ way- ANOVA; $n=3$ each).

Representative images of transwell chambers shown in bottom panel. d Tumor growth curves illustrating the failure of HBEC-iKRASG12D tumor formation in the absence of KRASG12D induction [Off Dox; GFP (Orange), GATAD2B (Black)], rapid tumor formation in GATAD2B (Red) vs. GFP control (Blue) in the presence of Dox, and the ability to toggle KRAS expression from On to Off (On > Off) to demonstrate requirement of KRAS for tumor maintenance in GATAD2B (Purple) vs. GFP control (Green) tumors. e Confirmation of human GATAD2B overexpression (left, $200 \mu \mathrm{m}$ scale bar) and enhanced KI67 staining (right, $100 \mu \mathrm{m}$ scale bar) via immunohistochemicial analysis of HBEC-iKRASG12D SQ tumors expressing GATAD2B vs. GFP from d. f Number of barcode sequencing reads from metastatic tissues (lymph node, lung) isolated from mice harboring HBEC-iKRASG12D_GATAD2B SQ tumors (On Dox) from d. NGS barcode sequencing was performed to quantitate presence of GATAD2B-specific barcode. Note that no metastases were observed in mice harboring iKRASG12D-GATAD2B SQ tumors, thus barcode sequencing was not performed. $\mathbf{g}$ Anchorage-independent colony formation assays for KRAS mutant (H23, A549, CALU-1) and KRAS wild-type (H1437, H1568) NSCLC cells $(N=3)$. shNT = Non-targeting control 
Therefore, we leveraged a well-established GEM model of lung adenocarcinoma where tumor initiation is achieved by Cre recombinase-mediated activation of a KRAS ${ }^{G 12 D}$ allele (hereafter $L S L-K R A S^{G 12 D ; 35}$. In addition to the use of lung-specific Cre driver alleles engineered into mice ${ }^{5-7,40}$, lung tumorigenesis in the LSL-KRASG12D GEM models can be achieved through application of adeno- or lenti-Cre virus through nasal inhalation or intubation ${ }^{41}$. To build on previous studies that have used this approach for the co-delivery of cDNAs or other genetic elements ${ }^{35,42}$, we devised a new lentiviral expression construct compatible with HiTMMoB that permits co-expression of barcoded ORFs with Cre recombinase (Fig. 4a). By crossing the LSL$K R A S^{G 12 D}$ mice with a strain carrying a Cre-inducible Luciferase allele [LSL-Luciferase ${ }^{43}$ ], we generated a mouse strain such that delivery of Cre recombinase permits co-expression of KRASG12D and Luciferase in the same cells (Fig. 4a). To test our lentiviral vector, we recombined ORFs encoding GFP and dominantnegative TP53 $\left(T P 53^{R 270 H}\right)$ into separate lentiviral constructs with unique barcodes, followed by virus titering using HEK293loxP-GFP-RFP Cre reporter cells. Intubation-mediated delivery of high titer virus (500,000 particles) encoding barcoded GFP- and

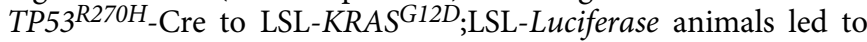
formation of lung tumors by 3 months as visualized by bioluminescent imaging (Fig. 4b). Animal necropsies confirmed numerous focal lesions throughout the lungs of animals intubated with either GFP-Cre or $T P 53^{R 270 H_{-}}$Cre (Fig. 4c). In contrast to mice exposed to GFP-Cre, those intubated with $T P 53^{R 270 H_{-}}$-Cre exhibited gross metastases to proximal and distant lymph nodes (Fig. 4c) consistent with other reports demonstrating metastatic activity of the TP53 ${ }^{R 270 H}$ allele in the $L S L-K R A S^{G 12 D}$ model $^{44}$. As expected, immunoblot analysis confirmed increased expression of TP53 in tumor-infiltrated lung and lymph node tissues from mice intubated with $T P 53^{R 270 H_{-}}$-Cre vs. mice who received GFPCre (Fig. 4e). NGS analysis of these tissues confirmed robust

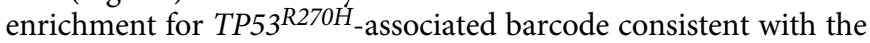
presence of $T P 53^{R 270 H_{-}}$Cre transduced cells (Fig. $4 \mathrm{f}$ ).

We next used this strategy to determine if elevated expression of GATAD2B would enhance tumorigenesis in the LSL-KRASG12D; LSL-Luciferase GEM model. Intubating animals with a reduced viral dose $(50,000$ particles) to achieve $<15 \%$ infection efficiency (based on optimization studies, see Methods) extended the overall latency of GFP-Cre infected mice as reported ${ }^{41}$ and resulted in weak Luciferase positivity up to 12 months post-infection, as assessed by serial bioluminescence imaging (Supplementary Fig. 6). In contrast, delivery of GATAD2B-Cre virus at the same dosage led to robust Luciferase signal at approximately 6 months (Fig. 4g). Animal necropsies confirmed marked tumor burden in GATAD2B-Cre infected mice (Fig. 4h), which was consistent with Luciferase imaging. Immunohistochemical analysis of lung tissue from tumor-bearing mice intubated with GATAD2B-Cre virus confirmed strong GATAD2B expression and Ki-67 positive nuclei (Fig. 4h), and immunoblot analysis confirmed elevated levels of GATAD2B protein in the lungs of mice intubated with GATAD2B-Cre virus compared to GFP-Cre control virus (Fig. 4i). These data corroborate our results using the HBEC model, indicating that expression of GATAD2B in the context of activated $K R A S$ promotes accelerated lung cancer progression.

GATAD2B cooperates with MYC to promote tumor progression. We next sought to identify the molecular mechanism by which GATAD2B exerts its pro-oncogenic and metastatic activities. For this we performed OncoPPi screening to map potential oncogenic GATAD2B interactors using a lung-cancer associated gene library ${ }^{45}$, and a highly efficient protein-protein interaction (PPI) detection platform derived from BRET ${ }^{\mathrm{n}}$ technology ${ }^{46,47}$.
The Nanoluc-tagged GATAD2B, serving as BRET $^{\mathrm{n}}$ donor, was scanned across 83 lung-cancer associated genes fused with Venus-tag ${ }^{45}$, serving as BRET $^{\mathrm{n}}$ acceptor, in a multiple DNA titration combination fashion to generate BRET ${ }^{n}$ saturation curve. The stringent proximity requirement $(<10 \mathrm{~nm})$ for efficient energy transfer from donor to acceptor allows the detection of protein partners to which GATAD2B directly binds. Statistical analysis of BRET signal, protein expression signal and BRETn saturation curves were performed to define positive PPIs with fold-over control of the area under curve $\left(\mathrm{FOC}_{\mathrm{AUC}}\right)>1.0, p<0.01$ $t$-test. The GATAD2B-MYC pair showed the strongest BRET ${ }^{\mathrm{n}}$ signal $\left(\mathrm{FOC}_{\mathrm{AUC}}>8, p<0.001, t\right.$-test) and typical $\mathrm{BRET}^{\mathrm{n}}$ saturation curve with protein expression dependent increase of BRET signal (Fig. 5a). We further confirmed the interaction between GATAD2B and MYC with conventional GST-pull down. Indeed, Venus-flag-MYC was detected in complex with GST-GATAD2B (Fig. 5b). The observation of the GATAD2B/MYC PPI strongly suggests a potential role for MYC-driven oncogenicity in GATAD2B-driven metastatic lung-cancer with KRAS mutations.

Increased MYC activity has been previously identified across multiple cancer types, including $K R A S^{G 12 D_{\text {-driven }} \text { tumors where }}$ MYC has been shown to enhance disease progression ${ }^{28,48}$. Interestingly, MYC was among the 28 ORFs found positively enriched in metastases isolated from our primary screen (Fig. 2a), thereby further supporting its role in lung cancer metastasis. We hypothesized that the physical association of GATAD2B with MYC could modulate expression of MYC-regulated transcripts, thus we examined the effect of GATAD2B expression on the activity of a MYC reporter construct containing three (GCCACGTGGCCACGTGGCCACGTGGC) wild type or mutated (GCCTCGAGGCCTCGAGGCCTCGAGGC) MYCbinding E-box elements upstream of a Luciferase coding sequence. Co-transfection of HEK293T cells with GATAD2B and the MYC reporter construct led to a 10 -fold increase of Luciferase activity compared to vector control $(p<0.001 t$-test); (Fig. 5c). Importantly, this GATAD2B-mediated increase in reporter activity was attenuated upon mutation of the E-box binding elements (Fig. 5c), indicating that GATAD2B activity requires the ability of MYC to bind to its target. Our hypothesis that GATAD2B modulates expression of MYC target genes is further supported by transcriptome analysis of the HBEC$i K R A S^{G 12 D}$ cells stably transduced with GATAD2B, where induction of $K R A S^{G 12 D}$ with Dox led to significant transcriptional modulation of hallmark MYC-regulated targets (SRSF1, HSPD1, HSPE1) as determined by MSigDB ( $p>5.88 \mathrm{e}-12$, FDR 7.35e-11, Supplementary Table 3, Fig. 5d). Similarly, analysis of the HBEC$i K R A S^{G 12 D}$;GATAD2B tumor protein lysates by Reverse Phase Proteomics Array (RPPA) compared to small, arrested GFP tumors showed elevated protein levels consistent with three previously published reports of MYC target pathway activation confirmed by chromatin immunoprecipitation ${ }^{49}$, ChIP on chip of cell lines identifying high-affinity MYC-binding ${ }^{50}$, or identified from the Pathway Interaction Database ${ }^{51}$ (Supplementary Table 3, Supplementary Fig. 7, Fig. 5e).

To examine whether MYC is required for GATAD2B-driven tumor growth in the context of Dox-regulated KRASG12D activation as in Fig. 3d, we used validated shRNA hairpin to knockdown MYC (Supplementary Fig. 8) in the HBEC-iKRAS cells overexpressing GATAD2B. Compared to mice with subcutaneously implanted HBEC-iKRAS cells treated with nontargeting shRNA and maintained on a Dox diet, MYC knockdown significantly decreased tumor formation and eliminated visible metastases (Fig. 5f). Together, these data indicate that GATAD2B physically associates with MYC and that MYC is required for GATAD2B-dependent tumor growth in KRAS G12D expressing cells. 
a

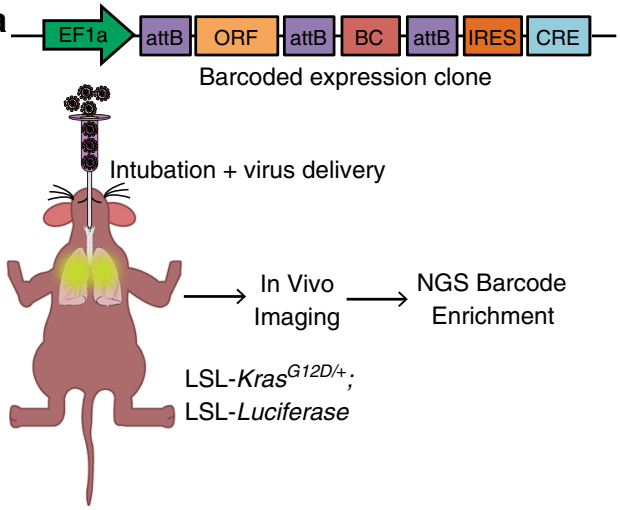

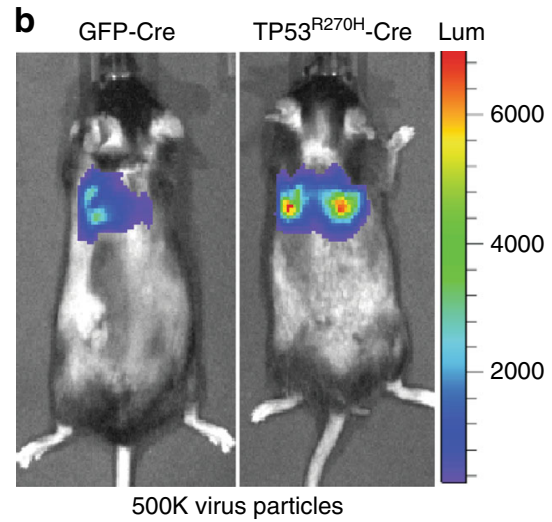
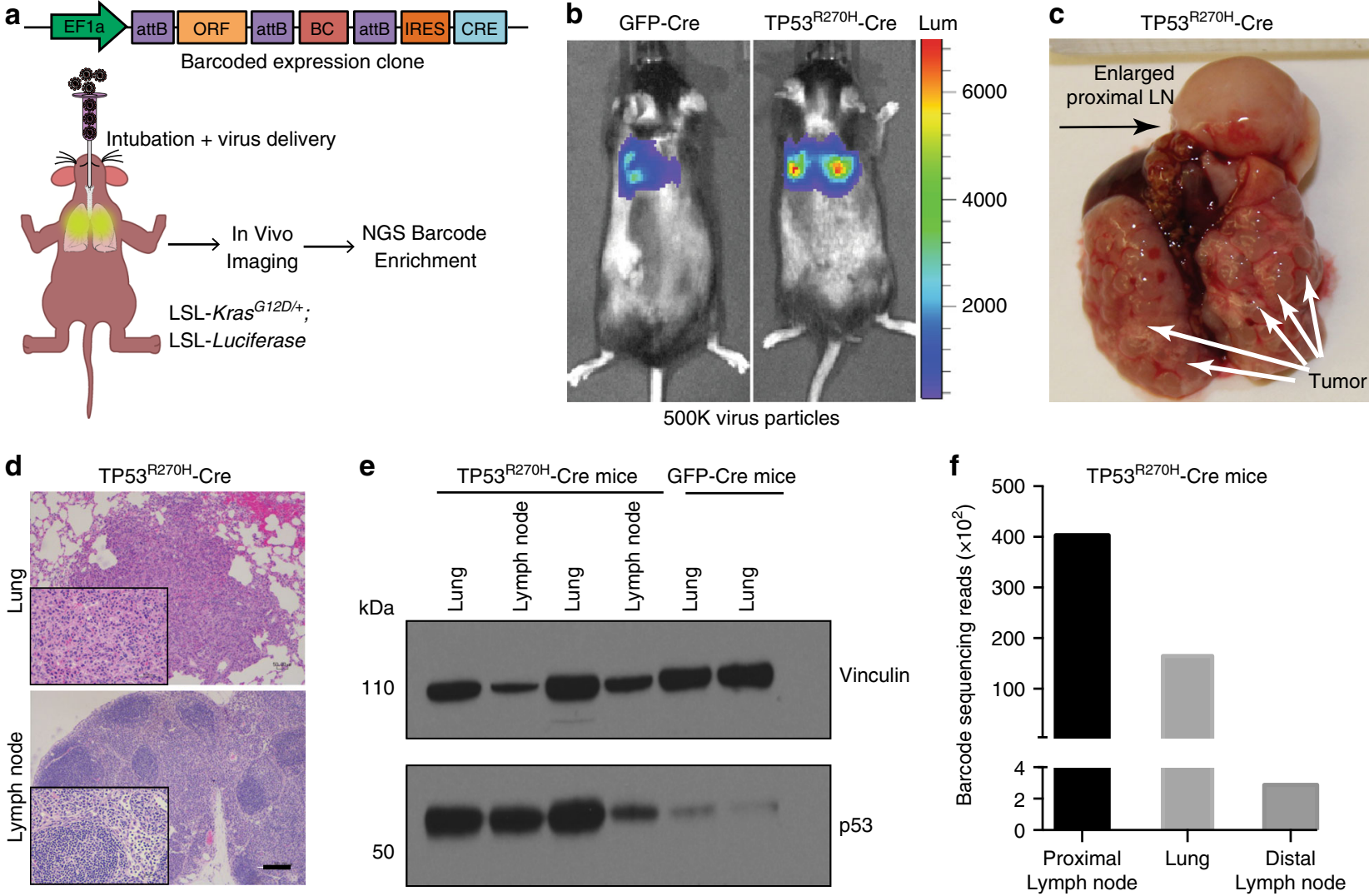

g
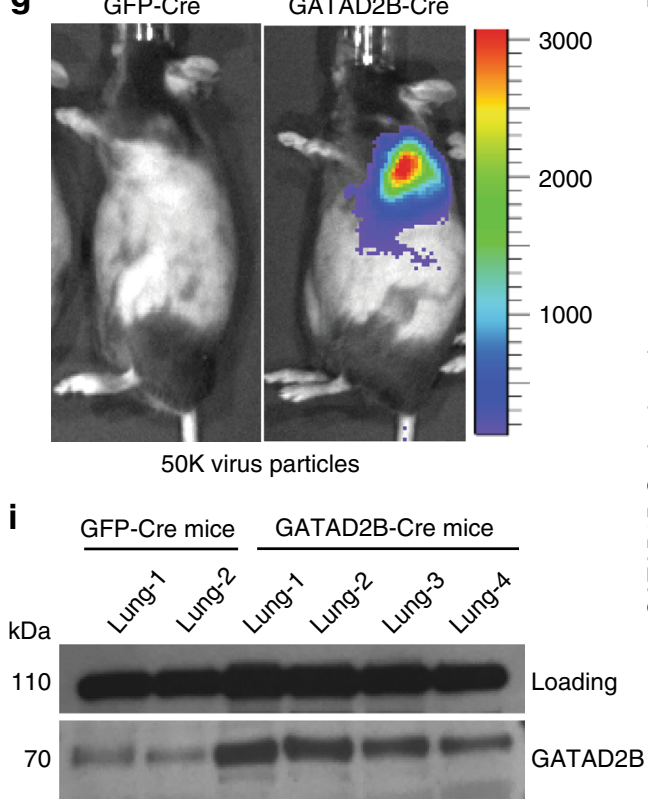

h
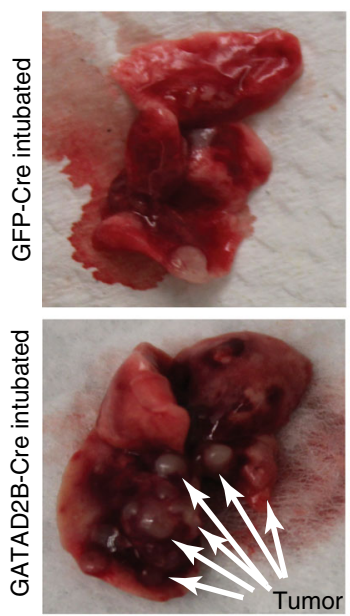

$\mathrm{H} \& \mathrm{E}$

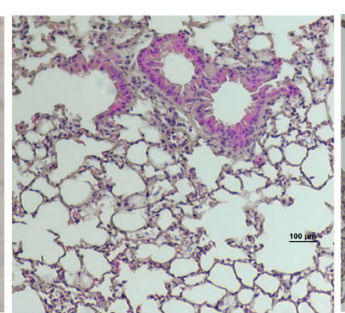

KI67

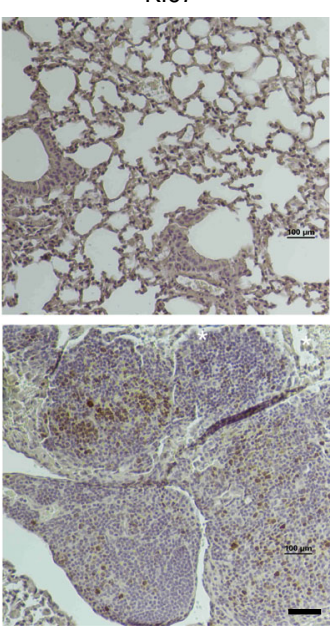

Fig. 4 GATAD2B enhances primary lung cancer progression. a Schematic illustration of primary tumor and metastasis tracking with ORF-Cre lentivirus. Expression of GATAD2B or GFP is driven by EFla promoter and assigned unique 24 nucleotide DNA barcode with downstream IRES element and Cre

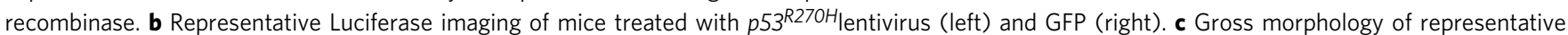
p53 $3^{\mathrm{R} 270 \mathrm{H}}$ driven lung tumors and metastasis to proximal Lymph Node. $\mathbf{d}$ Representative H\&E stains of resulting tumors in lung and lymph node tissue of p53 ${ }^{\mathrm{R} 270 \mathrm{H}}$ treated mice ( $50 \mu \mathrm{m}$ scale bar). e p53 Immunoblot in lung and lymph node tissues of mice intubated with p53 $270 \mathrm{H}$-Cre lentivirus or GFP-Cre lentivirus. $\mathbf{f}$ Number of barcode sequencing reads from metastatic tissues (lymph node, lung) isolated from $p 53^{R 270 H}$ mice. $\mathbf{g}$ Representative Luciferase imaging of mice treated with GATAD2B lentivirus (left) and GFP (right). h Representative gross morphology, H\&E stains, and positive nuclei stains of the $\mathrm{Ki}-67$ proliferation marker of resulting tumors in GATAD2B treated mice (bottom) vs. GFP (top). $\mathbf{i}$ Immunoblotting for GATAD2B in lung tissues in mice intubated with GATAD2B-Cre lentivirus or GFP-Cre lentivirus 
a

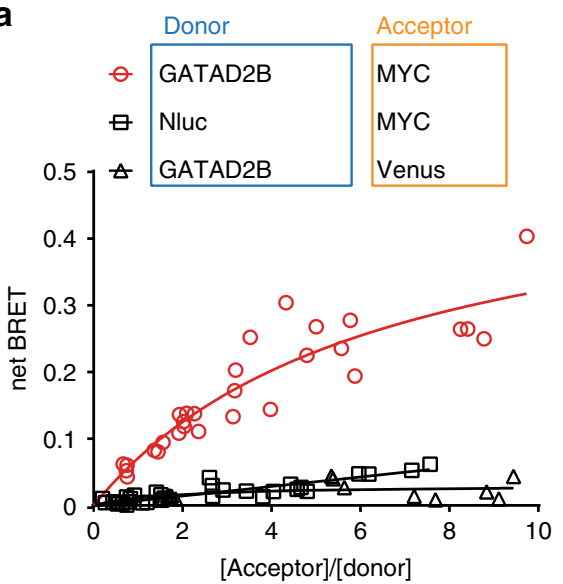

C

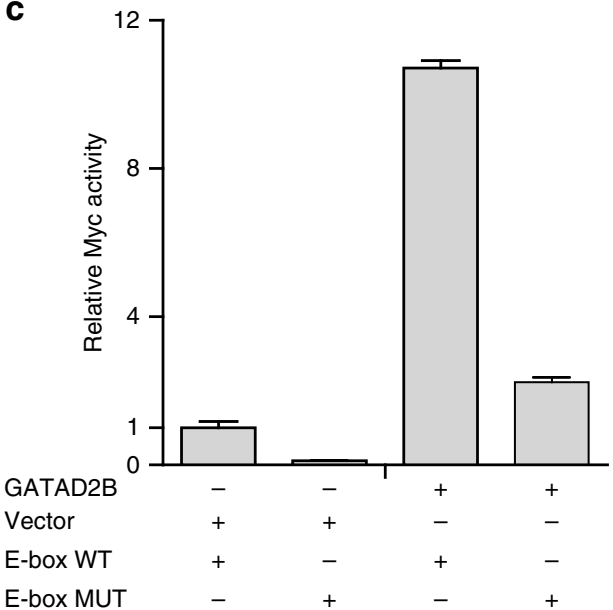

e

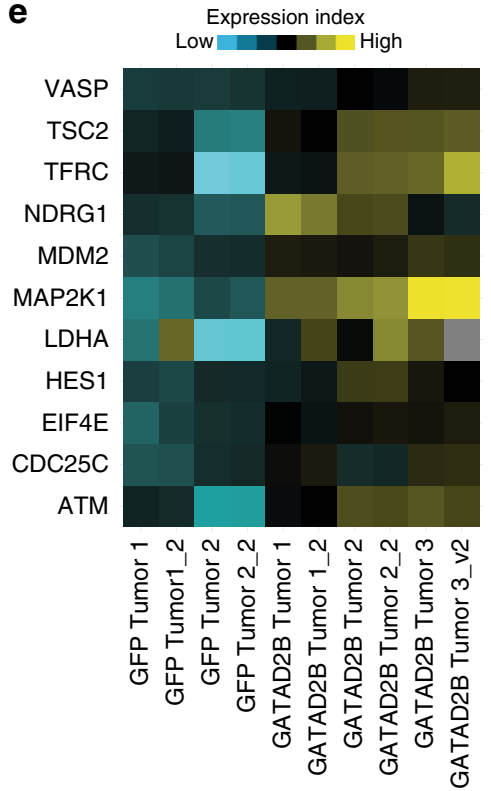

b

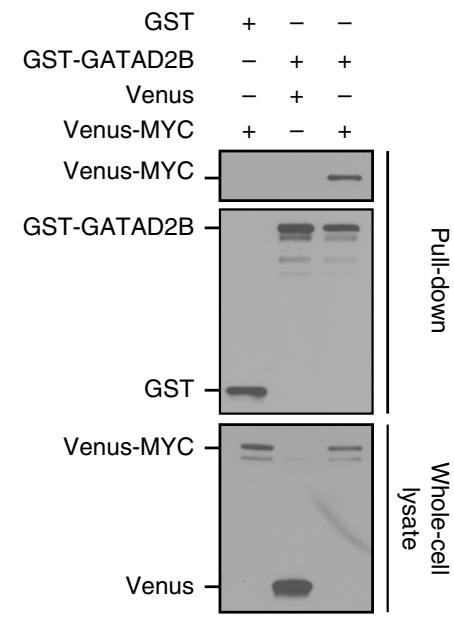

d

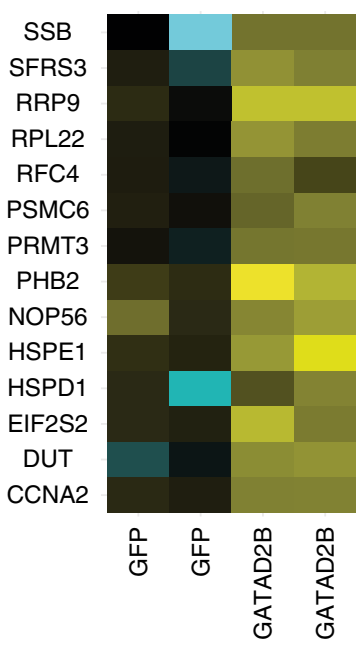

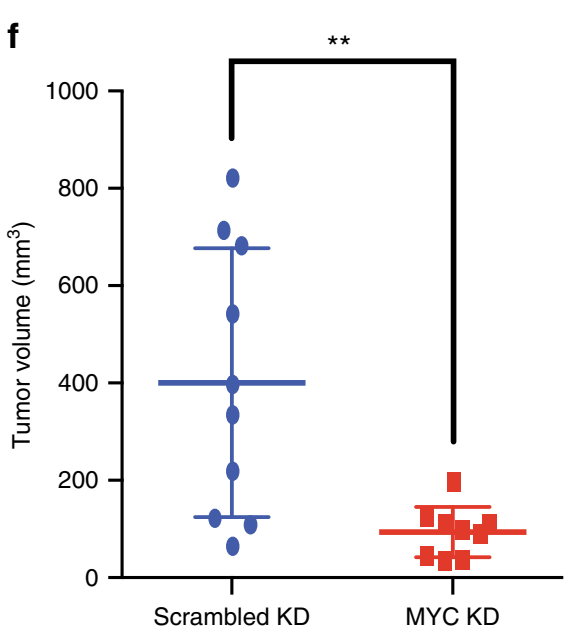

Fig. 5 GATAD2B interacts with C-MYC to enhance KRAS driven tumor growth. a BRET ${ }^{\mathrm{n}}$ saturation curve of GATAD2B-MYC interaction identified from OncoPPi screen. b GST-pull down validation of GATAD2B-MYC interaction. c MYC-reporter assay revealing the GATAD2B-induced increased MYC transcriptional activity. Mutated E-box was used as negative control. d Differentially expressed MYC-regulated mRNAs (hallmark MYC targets, MSigDB, $p<5.88 \mathrm{E}-12$, FDR $q<7.35 \mathrm{E}-11)$ in GATAD2B vs GFP KRASG12D activated HBEC cells. e Differentially expressed proteins of direct MYC-target genes in GATAD2B- $(N=6)$ and GFP-driven tumors $(N=4)$. f End point tumor volume analysis ( ${ }^{\star \star} p<.0057$, Mann-Whitney $\left.U\right)$ of GATAD2B-overexpressing HBEC line with MYC knockdown $(n=9)$ compared to scrambled control $(n=10)$ 


\section{Discussion}

Metastatic disease remains an elusive target for cancer treatment and remains the primary event defining outcome during the course of treatment for lung cancer patients. Despite major improvements made with early detection, and increased knowledge and identification of actionable tumor biomarkers, KRASdriven lung adenocarcinoma still represents a commonly diagnosed form of NSCLC for which there are no targeted agents. Therefore, the identification of genes that work in concert with $K R A S$ to drive tumor progression and/or metastasis provide the greatest hope of improving patient outcomes through personalized treatment options, either by acting as a direct target or targeting converging signaling pathways.

Here we performed an in vivo functional screen of 217 genetic aberrations identified from lung cancer genomics datasets. Our primary screen generated a functionally annotated list of 28 genes that led to robust metastatic dissemination and merit further interrogation, including some that have been previously identified to be mutated in lung adenocarcinoma (GNAS, MAPK6, $A C V R 1 B, F B X W 7, N T R K 3)$. While this functional screening strategy has identified genetic aberrations with in vivo metastatic ability, individual functional assessment of genes scoring as metastasis drivers requires validation with additional evidence to robustly delineate their roles in the malignant phenotypes and underlying mechanisms of action. The newly developed tools outlined here allowed us to demonstrate the context-specific transformative capabilities of $G A T A D 2 B$ and will serve for the study of other genes that may be dependent on KRAS comutation.

GATAD2B is a member of the Nucleosome Remodeling complex (NuRD), 1 of 4 major ATP dependent chromatin remodeling complexes ${ }^{52,53}$. The major roles of the complex are regulation of transcription, chromatin assembly, cell cycle progression, and genomic stability ${ }^{27}$. The NuRD complex primarily functions through recruitment of HDACs to discrete loci and has been previously implicated in tumor progression and metastasis through the complex members, metastasis-associated proteins 1 and 2 (MTA1 and MTA2) $38,54-56$. Despite these known roles of the Nucleosome Remodeling and Deacetylating (NuRD) complex in driving metastatic activity, GATAD2B has not been previously implicated in the metastatic capabilities of this complex. To date, GATAD2A and GATAD2B have been demonstrated to be structural components that bind to histone tails ${ }^{28}$.

We demonstrate that GATAD2B enhances tumor growth, at least in part, through a direct interaction with MYC, a known potent oncogenic and metastasis driver that also scored in the in vivo screen. Poor prognostic outcomes for patients harboring mutant KRAS and MYC amplification have been previously reported in human cancers ${ }^{9,57}$. In human bronchial epithelial cell (HBEC) models, MYC greatly enhanced aggressiveness of KRAS neoplastic transformation and induction of epithelial-tomesenchymal transition (EMT) $)^{58,59}$. In addition to human studies correlating elevated MYC levels with worse clinical outcomes, disease progression, and metastasis ${ }^{48,60,61}$, in animal models, spontaneous metastatic spread and rapid tumor progression is observed in KRAS mutant tumors with exogenous MYC 62,63 .

We identified activation of downstream MYC target genes in vitro and in vivo in GATAD2B-driven tumors. This is an intriguing finding because NURD activity has not been previously linked with physical interactions to oncogenic c-MYC, although the MTA1 subunit of the NURD complex has been shown to be a transcriptional target of $\mathrm{c}-\mathrm{MYC}$, required for Myc-induced transformation of cells ${ }^{55}$. This finding suggests an important level of whole-genome epigenetic reprogramming that is required for the progression and metastasis of KRAS-mutant lung cancer.
The identification of convergence between KRAS signaling and NURD/MYC epigenetic reprogramming may inform evidencebased clinical trial development for metastatic lung cancer patients with activated KRAS mutations, or even potentially other tumor types with KRAS mutations. Given that KRAS and MYC are currently therapeutically undruggable genetic alterations found in many tumor types, a greater understanding of the interaction and cooperativity between these oncogenic changes and GATAD2B may open up the exploration of new therapeutic approaches. Analysis of primary tumors in our study showed elevated signaling pathways commonly shared between tumor growth drivers and metastasis-inducing genes. These include HIF1A and hypoxia ${ }^{64}$, MET pathway activation ${ }^{65}$ and mTOR signaling ${ }^{66}$. Further in-depth study will be needed to understand what role these pathways play in the malignant phenotypes we observed. This study describes a functional screening platform that can readily identify novel candidates, several of which have been validated herein or in the companion paper on TMEM106 $\mathrm{B}^{67}$, and which are poised for future studies. More studies are warranted to determine whether GATAD2B may be targeted therapeutically, and given the role of GATAD2B as a NuRD complex member, emerging therapeutic options such as selective HDAC or BRD4 inhibitor based-strategies may be warranted.

\section{Methods}

Gene candidate selection. We utilized data collected from GEM models with a latent, somatically activated Kras allele in a mutant $p 53$ background (Kras ${ }^{G 12 D}$, $\left.p 53^{R 172 H \Delta G}\right)$. Mice harboring these mutations develop metastatic lung adenocarcinoma ${ }^{68}$, the most common histological subtype of non-small cell lung cancer (NSCLC), and the combination of Kras G12D and mutant $p 53$ is frequently found in human NSCLC ${ }^{24}$. This GEM model was used to derive a panel of lung tumor cell lines confirmed to be heterozygous for both the $p 53^{R 172 H \Delta G}$ and Kras ${ }^{G 12 D}$ alleles, and detailed characterization of these lines confirmed molecular and physiological properties that mirror human NSCLC ${ }^{18}$. Gene expression patterns of the $p 53^{R 172 H \Delta G}$, Kras $^{G 12 D}$ tumor explants using the metastasis-incompetent $393 \mathrm{P}$ cell line as reference revealed 3000 genes were differentially expressed (fold-change increase or decrease $>1.5 ; p<0.01$, paired $t$-test) in metastasis capable $393 \mathrm{LN}$ and 344 SQ tumors ${ }^{17}$. Overlapping genes found up-regulated in both comparisons were next triangulated with human copy number amplifications documented by TCGA (1.5-fold somatic amplification across $\geq 5 \%$ of 154 analyzed lung adenocarcinoma specimens). Additional genes with identified hyperactivating mutations were integrated ${ }^{24}$ that focused on 623 genes with potential relationships to cancer. Filtering copy number amplifications from TCGA with the 1013 non-synonymous somatic mutations identified by their study revealed 31 amplified genes that contain at least one validated missense mutation within the 188 analyzed tumors.

Cell culture. All cell lines were propagated at $37^{\circ} \mathrm{C}$ and $5 \% \mathrm{CO}_{2}$ in humidified atmosphere. $393 \mathrm{P}$ cells were published previously ${ }^{18}$ and were cultured in RPMI media supplemented with $10 \%$ Fetal Bovine Serum, 1\%Penicillin/Streptomycin. HBEC cells were a gift from J. Minna (University of Texas-Southwestern Medical center, see ${ }^{35}$ and were cultured in KSFM medium (Life Technologies). NSCLC cell lines were purchased from ATCC and cultured following their recommendations. In some experiments as indicated, cells were propagated in Doxycycline (500 ng/ml). The Dox-regulatable KRAS ${ }^{G 12 D}$ HBEC cell line was constructed using the pInducer system ${ }^{69}$ and constructed as described for HPDE-iKRAS ${ }^{G 12 D}$ cells previously ${ }^{11}$

In vivo screen and barcode enrichment analysis. For in vivo screening, pools of 20 cell lines were prepared with 50,000 cells per virally infected line containing 19 cell lines expressing individual ORFs and 1 expressing an mCherry control. These cells were mixed and re-suspended in RPMI solution without serum and Matrigel (BD Bioscience) for SQ implantation into $129 \mathrm{~Sv}$ at 1 million cells per site.

Barcode sequencing. gDNA was extracted from library-infected $393 \mathrm{P}$ cells (injected or Input) and individual tumor cores (output) for quadruplicate and triplicate, respectively, as done previously ${ }^{11}$. Barcode enrichment was assessed by quantitating the number of occurrences for each barcode sequence as a ratio to total number of barcode reads in each sample. Standard deviations were calculated for duplicate and triplicate reactions (where available) for input and output samples. 
NSCLC cell line assays. Soft agar assays were performed in 6-well plates in triplicate. First, bottom layers were prepared at $0.8 \%$ Noble agar (Affymetrix, Inc.) with complete RPMI growth medium. After solidification, 1000 cells were mixed with $0.45 \%$ agar in complete growth medium and laid on top of the bottom layer. Two milliliters medium was added in each well after 3 days and medium was refreshed every 3 days. Colonies were counted $4-5$ weeks after seeding. For knockdown studies, GATAD2B pLKO shRNAs were purchased from Thermo (TRCN0000230881, TRCN0000015315, TRCN0000230880), MYC shRNA (V2LHS_152051, Dharmacon) and used to transduce cells following manufacture's recommendations. Cell invasion assays were done as previously described $^{25}$. For cell proliferation assays, transduced HBECs cells were pretreated with Dox $(500 \mathrm{ng} / \mathrm{ml})$ for $48 \mathrm{~h}$, followed by plating 1000 cells onto White Opaque 96-well microplates in quadruplicate, respectively. Cell proliferation was assayed by CellTiter-Glo ${ }^{\circledR}$ (Promega) using a Wallac Victor2 Multilabel Counter at multiple time intervals, according to manufacturer instructions and previously ${ }^{11}$. Cells were stained for senescence-associated $\beta$-galactosidase activity according to manufacturer's instructions (Cell Signaling Technology). All data were assessed by two-tailed $t$-test calculation using Prism 6 , error bars represent s.e. m. (Graphpad).

Animal studies. All studies using mice were performed in accordance with our IACUC-approved animal protocols at Baylor College of Medicine and The University of Texas at MD Anderson Cancer Center. For xenograft tumor assays, $10^{5}$ cells were re-suspended in a 1:1 Hank's balanced salt solution (Life Technologies) and Matrigel (BD Biosciences) and injected into female athymic mice subcutaneously at bilateral flanks. Mice were monitored twice a week and tumors were measured and calculated by length $\times$ width $^{2} / 2$. Lung intubation studies were performed as described previously ${ }^{41}$. Lentivirus was produced using standard virus packaging vectors and virus protocols. Virus was filtered with $0.45 \mu \mathrm{m}$ filter followed by ultracentrifugation at 25,000 RPM for $3 \mathrm{~h}$ and resuspended in Hank's Balanced Salt Solution (HBSS). Cells were titered by transducing a Cre reporter cell line (amsbio SC018-Bsd) with 15\% transduction efficiency (for optimal MOI = 1). Subsequent luciferase imaging was completed with D-Luciferin (Gold Biotechnology) using the IVIS Lumina II. Xenograft studies for MYC knockdown experiment were performed as described earlier, pGIPZ MYC shRNAs (Clone ID: V2LHS_152051, Dharmacon) prepared following manufacture's recommendations cells were suspended in HBSS at $2.4 \times 10^{5}$ / injection and combined in a 1:1 ratio with matrigel matrix (BD).

Immunoblotting and quantitative RT-PCR. Cells and tumor tissues were lysed with RIPA buffer with protease inhibitors cocktail (Sigma Aldrich) and phosphatase inhibitors cocktail (Calbiochem). Protein lysates were separated on 4-12\% BisTris gel (Life Technologies) and transferred to PVDF membranes. The following antibodies were used to detect protein expression: GATAD2B (Sigma Aldrich 1:1000), Vinculin (Cell Signaling Technologies 1:1000), Erk1/2 (Cell Signaling Technologies 1:1000) Vinculin (CST, 1:10000) and GAPDH (Santa Cruz 1:25,000). Uncropped blots are available in the supplementary files.

Immunohistochemistry. Murine lung tissues or subcutaneous tumors were excised, washed in PBS, and fixed in formalin for $48 \mathrm{~h}$. Once fixed, samples were dehydrated in graded ethanol series followed by xylene. Samples were then embedded in paraffin, sectioned onto slides ( $5 \mathrm{~mm}$ thick), and allowed to dry. For $\mathrm{H} \& \mathrm{E}$ staining, slides were deparaffinized using standard procedure (Xylene $\times 2$ washes, $100 \%$ ethanol $\times 2$, 95\% ethanol, $70 \%$ ethanol, $50 \%$ ethanol), and stained with H\&E (Vector H-3401; Sigma HT110132). For immunohistochemical staining, slides were processed using Vectastain Elite ABC HRP Kit and DAB Peroxidase Substrate Kit (Vector Laboratories, PK-6101, SK-4100). Briefly, after deparaffinizing procedure (as per above), antigen retrieval was completed using $0.01 \mathrm{M}$ sodium citrate buffer ( $\mathrm{pH}$ 6.0), for $15 \mathrm{~min}$ at $95 \mathrm{C}$, followed by blocking with $0.3 \%$ $\mathrm{H} 2 \mathrm{O} 2$ for $30 \mathrm{~min}$. Slides were stained for GATAD2B antibody (1:5000, Sigma, HPA-017015) or Ki-67 antibody (1:500, Cell Signaling, 9027, hum; 1:500, Abcam, ab16667, mus) and processing and staining were completed using Vectastain and $\mathrm{DAB}$ kits in accordance with instructions provided by the manufacturer. qPCR primers were used as the following: (GATAD2B: F-5'-CAAAAGCTGTGCCTCA CTTC R-5'-TTCCAGTGAGGGGTGAAATC KRAS: F-5'-GGACTGGGGAGGC TTTCT R-5'-GCCTGTTTTGTGTCTACTGTTCT MYC: F-5' - CATCCTGTCCG TCCAAGCAG R-5' - CCTTACTTTTCCTTACGCACAAGA) qPCR was performed as previously described ${ }^{25}$.

GATAD2B interactors screening. A BRET ${ }^{\mathrm{n}}$ technology-based OncoPPi screening platform was used to scan GATAD2B across lung-cancer associated gene library ${ }^{4}$. Briefly, NLuc-tagged GATAD2B was co-transfected with Venus-tagged gene in pairwise into HEK293T cells seeded in 1536-well plate. Two sets of 1536-well plates, one white plate (Corning) for BRET signal measurement and another black plate (Corning) for the measurement of Venus fluorescence intensity, were prepared side-by-side as previously described ${ }^{46,47}$. Linear polyethylenimines (Polysciences) were used as transfection reagent throughout the study. 384-cannula array integrated with Sciclone ALH 3000 liquid handler (PerkinElmer) was used to assist high-throughput transfection. $48 \mathrm{~h}$ after transfection, Nano-Glo ${ }^{\circledast}$ luciferase substrate furimazine was added to the cells directly. PPI signal, NLuc-GATAD2B expression and Venus-genes expression were monitored by measuring BRET ratio, luminescence signal and fluorescence signal, respectively, using an Envision Multilabel plate reader (PerkinElmer). The BRET ratio is calculated as the ratio of intensity at $535 \mathrm{~nm}$ over $460 \mathrm{~nm}$. The net BRET is the bleed-through corrected BRET ratio. The relative amount of NLuc-GATAD2B and Venus-gene partners expressions were measured by the luminescence signal at $460 \mathrm{~nm}\left(\mathrm{~L}_{460}\right)$ and the laser-excited Venus fluorescence intensity (FI). The ratio of relative amount of acceptor over donor protein expression (Acceptor/Donor) was defined as Venus $\mathrm{FI} / \mathrm{L}_{460}$. Area under the BRET saturation curve (AUC) was built by fitting the net BRET on $Y$-axis and Acceptor/Donor on $X$-axis using one-site binding equation, and the statistical significance was calculated with the unpaired two-tailed $t$-test as described previously 46,47

MYC reporter assay. MYC-luciferase reporter assay was conducted essentially as described previously 45,70 . HEK293T cells were transfected with Venus-FlagGATAD2B or Flag-Venus vector along with the Myc-E-box-containing Firefly luciferase reporter plasmid. Renilla luciferase expression vector served as a calibration control. The proteins were expressed in cells for $48 \mathrm{~h}$ in DMEM media supplemented with $10 \%$ FBS. Then, cells were harvested, and transferred to a 384-well plate $(20 \mu \mathrm{L}$ per well). MYC reporter assay was performed using DualGlo luciferase kit (Promega) following manufacturer's instructions. The normalized luminescence was calculated as a ratio of luminescence of Firefly luciferase to the luminescence of Renilla luciferase. The statistical significance was calculated with the unpaired two-tailed $t$-test.

Transcriptome profiling and RPPA. RNAs extracted from the indicated cell lines cultured with or without Dox ( $500 \mathrm{ng} \mathrm{ml}$ for $48 \mathrm{~h}$ ) were processed for hybridization on Illumina Human Bead Array by the Baylor College of Medicine Genome Profiling Core Facility. Array data were deposited in NCBI Gene Expression Omnibus (GEO) database (http://www.ncbi.nlm.nih.gov/geo/) under accession number GSE100336. RPPA was performed by the MD Anderson RPPA Core Facility using protein lysates extracted from the indicated subcutaneous tumors. For each RPPA replicate, cells were washed with cold PBS twice and lysed with $100 \mu \mathrm{l}$ lysis buffer ( $1 \%$ Triton X-100, $50 \mathrm{mM}$ HEPES, pH 7.4, $150 \mathrm{mM} \mathrm{NaCl}$, $1.5 \mathrm{mM} \mathrm{MgCl} 2,1 \mathrm{mM}$ EG/TA, $100 \mathrm{mM} \mathrm{NaF}, 10 \mathrm{mM}$ Na pyrophosphate, $1 \mathrm{mM}$ $\mathrm{Na} 3 \mathrm{VO} 4$ and $10 \%$ glycerol, containing freshly added protease and phosphatase inhibitors from Roche Applied Science Cat. \# 05056489001 and 04906837001 , respectively), mixed with $4 \times$ SDS sample buffer ( $40 \%$ Glycerol, $8 \%$ SDS, $0.25 \mathrm{M}$ Tris- $\mathrm{HCl}, \mathrm{pH}$ 6.8) and boiled for $5 \mathrm{~min}$ before RPPA. Two-sided homoscedastic $t$-tests (using log-transformed data) and fold changes were used to determine differentially expressed genes and proteins. For transcriptome data, gene probes significant with fold change $>1.5$ and $p<0.01$ Dox vs. no Dox for either KRAS WT or KRAS G12D cells were clustered using a "supervised" clustering approached as previously described ${ }^{71}$. Expression patterns were visualized as heatmaps using JavaTreeView or R statistical software. MSigDB (version 3.0) gene sets were searched using SigTerms ${ }^{72}$. Grubbs test was used to remove significant outliers where appropriate.

Data availability. Array data were deposited in NCBI Gene Expression Omnibus (GEO) database (http://www.ncbi.nlm.nih.gov/geo/) under accession number GSE100336. All other relevant data are available from the authors.

Received: 8 August 2017 Accepted: 2 May 2018

Published online: 16 July 2018

\section{References}

1. Siegel, R. L., Miller, K. D. \& Jemal, A. Cancer statistics, 2016. CA Cancer J. Clin. 66, 7-30 (2016)

2. Paez, J. G. et al. EGFR mutations in lung cancer: correlation with clinical response to gefitinib therapy. Science 304, 1497-1500 (2004).

3. Kwak, E. L. et al. Anaplastic lymphoma kinase inhibition in non-small-cell lung cancer. N. Engl. J. Med. 363, 1693-1703 (2010).

4. Imielinski, M. et al. Mapping the hallmarks of lung adenocarcinoma with massively parallel sequencing. Cell 150, 1107-1120 (2012).

5. Ehrhardt, A. et al. Development of pulmonary bronchiolo-alveolar adenocarcinomas in transgenic mice overexpressing murine c-myc and epidermal growth factor in alveolar type II pneumocytes. Br. J. Cancer 84, 813-818 (2001).

6. Meuwissen, R. \& Berns, A. Mouse models for human lung cancer. Genes Dev. 19, 643-664 (2005).

7. Jackson, E. L. et al. Analysis of lung tumor initiation and progression using conditional expression of oncogenic K-ras. Genes Dev. 15, 3243-3248 (2001). 
8. Skoulidis, F. et al. Co-occurring genomic alterations define major subsets of KRAS-mutant lung adenocarcinoma with distinct biology, immune profiles, and therapeutic vulnerabilities. Cancer Discov. 5, 860-877 (2015).

9. Cancer Genome Atlas Research, N. Comprehensive molecular profiling of lung adenocarcinoma. Nature 511, 543-550 (2014).

10. Dogruluk, T. et al. Identification of variant-specific functions of PIK3CA by rapid phenotyping of rare mutations. Cancer Res. 75, 5341-5354 (2015).

11. Tsang, Y. H. et al. Functional annotation of rare gene aberration drivers of pancreatic cancer. Nat. Commun. 7, 10500 (2016).

12. Scott, K. L. et al. Proinvasion metastasis drivers in early-stage melanoma are oncogenes. Cancer Cell. 20, 92-103 (2011).

13. Kim, M. et al. Comparative oncogenomics identifies NEDD9 as a melanoma metastasis gene. Cell 125, 1269-1281 (2006).

14. Zender, L. et al. Identification and validation of oncogenes in liver cancer using an integrative oncogenomic approach. Cell 125, 1253-1267 (2006).

15. Maser, R. S. et al. Chromosomally unstable mouse tumours have genomic alterations similar to diverse human cancers. Nature 447, 966-971 (2007).

16. Ding, Z. et al. SMAD4-dependent barrier constrains prostate cancer growth and metastatic progression. Nature 470, 269-273 (2011).

17. Gibbons, D. L. et al. Expression signatures of metastatic capacity in a genetic mouse model of lung adenocarcinoma. PLoS ONE 4, e5401 (2009).

18. Gibbons, D. L. et al. Contextual extracellular cues promote tumor cell EMT and metastasis by regulating miR-200 family expression. Genes Dev. 23, 2140-2151 (2009).

19. Shayesteh, L. et al. PIK3CA is implicated as an oncogene in ovarian cancer. Nat. Genet. 21, 99-102 (1999).

20. Samuels, Y. et al. High frequency of mutations of the PIK3CA gene in human cancers. Science 304, 554 (2004).

21. Osajima-Hakomori, Y. et al. Biological role of anaplastic lymphoma kinase in neuroblastoma. Am. J. Pathol. 167, 213-222 (2005).

22. George, R. E. et al. Activating mutations in ALK provide a therapeutic target in neuroblastoma. Nature 455, 975-978 (2008).

23. Mosse, Y. P. et al. Identification of ALK as a major familial neuroblastoma predisposition gene. Nature 455, 930-935 (2008)

24. Ding, L. et al. Somatic mutations affect key pathways in lung adenocarcinoma. Nature 455, 1069-1075 (2008).

25. Wardwell-Ozgo, J. et al. HOXA1 drives melanoma tumor growth and metastasis and elicits an invasion gene expression signature that prognosticates clinical outcome. Oncogene 33, 1017-1026 (2014).

26. Yang, X. et al. A public genome-scale lentiviral expression library of human ORFs. Nat. Methods 8, 659-661 (2011).

27. Lai, A. Y. \& Wade, P. A. Cancer biology and NuRD: a multifaceted chromatin remodelling complex. Nat. Rev. Cancer 11, 588-596 (2011).

28. Torchy, M. P., Hamiche, A. \& Klaholz, B. P. Structure and function insights into the NuRD chromatin remodeling complex. Cell. Mol. Life Sci. 72, 2491-2507 (2015).

29. Witkiewicz, A. K. et al. Whole-exome sequencing of pancreatic cancer defines genetic diversity and therapeutic targets. Nat. Commun. 6, 6744 (2015).

30. Biankin, A. V. et al. Pancreatic cancer genomes reveal aberrations in axon guidance pathway genes. Nature 491, 399-405 (2012).

31. Cerami, E. et al. The cBio cancer genomics portal: an open platform for exploring multidimensional cancer genomics data. Cancer Discov. 2, 401-404 (2012).

32. Gyorffy, B., Surowiak, P., Budczies, J. \& Lanczky, A. Online survival analysis software to assess the prognostic value of biomarkers using transcriptomic data in non-small-cell lung cancer. PLoS ONE 8, e82241 (2013).

33. Goswami, C. P. \& Nakshatri, H. PROGgeneV2: enhancements on the existing database. BMC Cancer 14, 970 (2014).

34. Rousseaux, S. et al. Ectopic activation of germline and placental genes identifies aggressive metastasis-prone lung cancers. Sci. Transl. Med. 5, 186ra166 (2013).

35. Meylan, E. et al. Requirement for NF-kappaB signalling in a mouse model of lung adenocarcinoma. Nature 462, 104-107 (2009).

36. Singh, A. et al. A gene expression signature associated with "K-Ras addiction" reveals regulators of EMT and tumor cell survival. Cancer Cell. 15, 489-500 (2009).

37. Denslow, S. A. \& Wade, P. A. The human Mi-2/NuRD complex and gene regulation. Oncogene 26, 5433-5438 (2007).

38. Fu, J. et al. The TWIST/Mi2/NuRD protein complex and its essential role in cancer metastasis. Cell Res. 21, 275-289 (2011).

39. Liang, J. et al. Nanog and Oct4 associate with unique transcriptional repression complexes in embryonic stem cells. Nat. Cell Biol. 10, 731-739 (2008).

40. Fisher, G. H. et al. Induction and apoptotic regression of lung adenocarcinomas by regulation of a K-Ras transgene in the presence and absence of tumor suppressor genes. Genes Dev. 15, 3249-3262 (2001).
41. DuPage, M., Dooley, A. L. \& Jacks, T. Conditional mouse lung cancer models using adenoviral or lentiviral delivery of Cre recombinase. Nat. Protoc. 4, 1064-1072 (2009)

42. Xia, Y. et al. Reduced cell proliferation by IKK2 depletion in a mouse lungcancer model. Nat. Cell Biol. 14, 257-265 (2012).

43. Safran, M. et al. Mouse reporter strain for noninvasive bioluminescent imaging of cells that have undergone Cre-mediated recombination. Mol. Imaging 2, 297-302 (2003).

44. Jackson, E. L. et al. The differential effects of mutant p53 alleles on advanced murine lung cancer. Cancer Res. 65, 10280-10288 (2005).

45. Li, Z. et al. The OncoPPi network of cancer-focused protein-protein interactions to inform biological insights and therapeutic strategies. Nat. Commun. 8, 14356 (2017).

46. Mo, X. L. et al. Enabling systematic interrogation of protein-protein interactions in live cells with a versatile ultra-high-throughput biosensor platform. J. Mol. Cell Biol. 8, 271-281 (2016).

47. Mo, X. L. \& Fu, H. BRET: NanoLuc-based bioluminescence resonance energy transfer platform to monitor protein-protein interactions in live cells. Methods Mol. Biol. 1439, 263-271 (2016).

48. Ischenko, I., Zhi, J., Moll, U. M., Nemajerova, A. \& Petrenko, O. Direct reprogramming by oncogenic Ras and Myc. Proc. Natl Acad. Sci. USA 110, 3937-3942 (2013).

49. Zeller, K. I., Jegga, A. G., Aronow, B. J., O’Donnell, K. A. \& Dang, C. V. An integrated database of genes responsive to the Myc oncogenic transcription factor: identification of direct genomic targets. Genome Biol. $\mathbf{4}$, R69 (2003).

50. Ben-Porath, I. et al. An embryonic stem cell-like gene expression signature in poorly differentiated aggressive human tumors. Nat. Genet. 40, 499-507 (2008).

51. Schaefer, C. F. et al. PID: the Pathway Interaction Database. Nucleic Acids Res. 37, D674-D679 (2009).

52. Brackertz, M., Boeke, J., Zhang, R. \& Renkawitz, R. Two highly related p66 proteins comprise a new family of potent transcriptional repressors interacting with MBD2 and MBD3. J. Biol. Chem. 277, 40958-40966 (2002).

53. Brackertz, M., Gong, Z., Leers, J. \& Renkawitz, R. p66alpha and p66beta of the Mi-2/NuRD complex mediate MBD2 and histone interaction. Nucleic Acids Res. 34, 397-406 (2006).

54. Nicolson, G. L. et al. Tumor metastasis-associated human MTA1 gene and its MTA1 protein product: role in epithelial cancer cell invasion, proliferation and nuclear regulation. Clin. Exp. Metastasis. 20, 19-24 (2003).

55. Zhang, X. Y. et al. Metastasis-associated protein 1 (MTA1) is an essential downstream effector of the c-MYC oncoprotein. Proc. Natl Acad. Sci. USA 102, 13968-13973 (2005).

56. Pencil, S. D., Toh, Y. \& Nicolson, G. L. Candidate metastasis-associated genes of the rat 13762NF mammary adenocarcinoma. Breast Cancer Res. Treat. 25, 165-174 (1993).

57. Pereira, C. B. et al. Prognostic and predictive significance of MYC and KRAS alterations in breast cancer from women treated with neoadjuvant chemotherapy. PLoS ONE 8, e60576 (2013).

58. Sato, M. et al. Human lung epithelial cells progressed to malignancy through specific oncogenic manipulations. Mol. Cancer Res. 11, 638-650 (2013).

59. Larsen, J. E. et al. ZEB1 drives epithelial-to-mesenchymal transition in lung cancer. J. Clin. Invest. 126, 3219-3235 (2016).

60. Volm, M., Drings, P., Wodrich, W. \& van Kaick, G. Expression of oncoproteins in primary human non-small cell lung cancer and incidence of metastases. Clin. Exp. Metastas. 11, 325-329 (1993).

61. Han, N. et al. Progressive lung cancer determined by expression profiling and transcriptional regulation. Int. J. Oncol. 41, 242-252 (2012).

62. Rapp, U. R. et al. MYC is a metastasis gene for non-small-cell lung cancer. PLoS ONE 4, e6029 (2009).

63. Podsypanina, K., Politi, K., Beverly, L. J. \& Varmus, H. E. Oncogene cooperation in tumor maintenance and tumor recurrence in mouse mammary tumors induced by Myc and mutant Kras. Proc. Natl Acad. Sci. USA 105, 5242-5247 (2008).

64. Devignes, C. S. et al. HIF signaling in osteoblast-lineage cells promotes systemic breast cancer growth and metastasis in mice. Proc. Natl Acad. Sci. USA 115, E992-E1001 (2018).

65. Tey, S. K. et al. Nuclear Met promotes hepatocellular carcinoma tumorigenesis and metastasis by upregulation of TAK1 and activation of NF-kappaB pathway. Cancer Lett. 411, 150-161 (2017).

66. Hsieh, A. C. et al. The translational landscape of mTOR signalling steers cancer initiation and metastasis. Nature 485, 55-61 (2012).

67. Kundu, S. T. et al. TMEM106B drives lung cancer metastasis by inducing TFEB-dependent lysosome synthesis and secretion of cathepsins. Nat. Commun.doi: 10.1038/s41467-018-05013-x (2018). 
68. Zheng, S., El-Naggar, A. K., Kim, E. S., Kurie, J. M. \& Lozano, G. A genetic mouse model for metastatic lung cancer with gender differences in survival Oncogene 26, 6896-6904 (2007).

69. Meerbrey, K. L. et al. The pINDUCER lentiviral toolkit for inducible RNA interference in vitro and in vivo. Proc. Natl Acad. Sci. USA 108, 3665-3670 (2011).

70. Ivanov, A. A. et al. OncoPPi-informed discovery of mitogen-activated protein kinase kinase 3 as a novel binding partner of c-Myc. Oncogene 36, 5852-5860 (2017).

71. Creighton, C. J. et al. Insulin-like growth factor-I activates gene transcription programs strongly associated with poor breast cancer prognosis. J. Clin. Oncol. 26, 4078-4085 (2008).

72. Creighton, C. J., Nagaraja, A. K., Hanash, S. M., Matzuk, M. M. \& Gunaratne, P. H. A bioinformatics tool for linking gene expression profiling results with public databases of microRNA target predictions. RNA 14, 2290-2296 (2008).

\section{Acknowledgements}

The authors would like to thank Dr. John Minna (UT Southwestern) for providing parental Human Bronchial Epithelial Cell lines. C.G. also wishes to thank Dr. Sharon Plon for her guidance and mentorship during this project and in review of this manuscript This project was supported in part by the Genomic and RNA Profiling Core at Baylor College of Medicine with funding from the NIH/NCI grant (P30CA125123) and Cytometry and Cell Sorting Core Facility with funding from the NIH (P30 AI036211, P30 CA125123, and S10 RR024574). This project was also supported by the Cancer Prevention and Research Institute of Texas (CPRIT; RP140216) by funding to K.L.S., the Department of Defense (LC110216) by funding to K..L.S. and D.LG., an MD Anderson Cancer Center Physician Scientist Award to D.L.G., and by the NIH (U01CA168394) by funding to K..L.S. and G.B.M. H. L. was supported by the CPRIT Pre-Doctoral Fellowship (RP140102). D.L.G. is a Lee Clark Fellow of the University of Texas MD Anderson Cancer Center. This project was also supported by the Cancer Target Discovery and Development Network grants U01CA168449 and U01CA217875 to H.F.

\section{Author contributions}

Conception and design: C.G., S.T.K., D.L.G. and K.L.S. Acquisition of data: C.G., S.T.K. O.Z., X.L.M., A.A.I., H.L., R.H.C., Y.H.T., D.M., M.M., K.E., J.J.F., S.A., F.C., Z.C. Analysis and interpretation of data: C.G., S.T.K., O.Z., F.C., K.C., C.J.C., D.L.G. and K.L.S. Writing, review, and/or revision of the manuscript: C.G. S.T.K., D.L.G. and K.L.S. Technical and material support: G.B.M. Study Supervision: H.F., D.L.G. and K.L.S

\section{Additional information}

Supplementary Information accompanies this paper at https://doi.org/10.1038/s41467 018-04572-3.

Competing interests: The authors declare no competing interests.

Reprints and permission information is available online at http://npg.nature.com/ reprintsandpermissions/

Publisher's note: Springer Nature remains neutral with regard to jurisdictional claims in published maps and institutional affiliations.

(c) Open Access This article is licensed under a Creative Commons Attribution 4.0 International License, which permits use, sharing, adaptation, distribution and reproduction in any medium or format, as long as you give appropriate credit to the original author(s) and the source, provide a link to the Creative Commons license, and indicate if changes were made. The images or other third party material in this article are included in the article's Creative Commons license, unless indicated otherwise in a credit line to the material. If material is not included in the article's Creative Commons license and your intended use is not permitted by statutory regulation or exceeds the permitted use, you will need to obtain permission directly from the copyright holder. To view a copy of this license, visit http://creativecommons.org/ licenses/by/4.0/.

(C) The Author(s) 2018 